\title{
Ciclopirox Olamine Induces Ferritinophagy and Ameliorates Disease Progression in Polycystic Kidney Disease
}

Priyanka S Radadiya, B.S. ${ }^{1,3,6}$, Rajni V Puri, Ph.D. ${ }^{1,3,6}$, Brenda Magenheimer, B.S. ${ }^{2,3,6}$, Dharmalingam Subramaniam, Ph.D. ${ }^{4,6}$, , Pamela V Tran, Ph.D. ${ }^{3,4,6}$, Hao Zhu, Ph.D. 5,6, Subhashini Bollisetty, Ph.D. ${ }^{7}$, James P Calvet, Ph.D.2,3,6, Darren P Wallace, Ph.D. ${ }^{1,3,6}$ and \# Madhulika Sharma, Ph.D. ${ }^{1,3,6}$

Departments of Internal Medicine ${ }^{1}$, Biochemistry and Molecular biology2, Anatomy and Cell biology ${ }^{4}$, Clinical Laboratory Sciences ${ }^{5}$, Department of Cancer Biology ${ }^{4}$, The Jared Grantham Kidney Institute ${ }^{3}$, University of Kansas Medical Center, Kansas City, Kansas ${ }^{6}$. Department of Internal Medicine, University of Alabama at Birmingham ${ }^{7}$

\section{\#Correspondence}

Madhulika Sharma, PhD

Department of Internal Medicine

University of Kansas Medical Center

Kansas City, KS 66160

Email: msharma3@kumc.edu

Phone: 9139459393 (office)

Fax: 9135889252

ORCD ID: https://orcid.org/0000-0001-7488-5444

\section{Sources of support}

K-INBRE summer scholarship award (P20 GM103418) to PR., the COBRE (COBRE, P30 GM12273) and the NIH R01 DK108433 to MS

\section{Running Title}


Ciclopirox ameliorates PKD in mice.

\section{Abstract}

Despite the recent launch of Tolvaptan, the search for safer polycystic kidney disease (PKD) drugs continues. Ciclopirox, as the free acid (CPX) and its olamine salt (CPX-O) , are contained in number of commercially available topical antifungal agents. CPX is reported in the literature to possess anticancer activity in number of solid tumor cancers and hematological malignancy by several proposed mechanisms of action including chelation of iron and inhibition of iron dependent enzymes. Here, we show that CPX-O inhibited in vitro cystogenesis of primary human cyst epithelial cells cultured in 3D collagen matrix. To determine if CPX-O inhibits PKD progression, we treated PKD mice with a low dose of 10 $\mathrm{mg} / \mathrm{kg}$ CPX-O by daily intraperitoneal injections from day 21 to day 49 postpartum. CPX-O reduced the kidney to body weight ratio of the PKD mice. This was associated with decreased cell proliferation decrease cystic area and improved renal function. We found that ferritin levels were significantly elevated in cystic kidneys of PKD mice, and that CPX-O treatment reduced renal ferritin levels and increased ferritinophagy marker, NCOA4. Our data suggest that CPXO dose dependently induces ferritin degradation via ferritinophagy which is associated with decreased cyst growth and disease progression in PKD mice. Most importantly these data indicate that CPX-O, a drug used to treat skin 
infections and currently in clinical trials for cancer, has the potential to treat ADPKD.

Key words: Ciclopirox olamine, ferritin, proliferation, polycystic kidney disease

\section{Introduction}

Polycystic kidney disease (PKD) is a genetic disease characterized by multiple cysts in the kidneys which enlarge overtime and lead to end stage renal disease. The incidence of autosomal dominant polycystic kidney disease (ADPKD) is 1 in every 1000 births. About $85 \%$ of ADPKD cases have mutations in polycystin 1 (PC1) and the remaining 15\% in polycystin 2 (PC2) (proteins encoded by PKD1 and $P K D 2$ respectively) ${ }^{1,2}$. Both PC1 and PC2 have been found to be localized to cilia. PC1 and PC2 function in the cilia and appear to be important in the regulation of intracellular $\mathrm{Ca}^{2+}$. Decreased intracellular $\mathrm{Ca}^{2+}$ levels in ADPKD cells in combination with activation of adenylate cyclase and accumulation of cAMP lead to increased proliferation and fluid secretion and contribute to enlargement of cysts ${ }^{3-6}$. Many signaling pathways have been shown to be deregulated in response to loss of function mutations in PKD $2,3,7,8$. 
We identified a drug Ciclopirox (CPX) or its olamine salt (CPX-O) as a drug candidate that could influence cyst progression as this drug has been shown to alter many pathways that are also affected in PKD such as the Notch signaling pathway, the mTOR pathway, the MEK-ERK pathway and the Wnt signaling pathway to name a few ${ }^{9-13}$. CPX is an antifungal, topical solution used in a variety of formulations such as gels, creams, shampoos for treatment of cutaneous fungal infections ${ }^{14}$. However, in last decade, the wide use of CPX in other diseases have been recognized ${ }^{15-17}$. Furthermore, the safe therapeutic response of CPX has been tested in various animals including pigs and dogs ${ }^{18}$. The preclinical studies have shown that systemic administration of CPX in hematologic malignancies resulted in antitumor effects ${ }^{19}$. The proposed mechanisms of CPX include chelation of polyvalent metal cations, such $\mathrm{Fe}+$ by which it can inhibit iron dependent enzymes, such as ribonucleotide reductase and components of hypoxia, Wnt, and Notch signaling ${ }^{10,20,21}$. CPX is also involved in ferritin degradation ${ }^{22}$ and protection against ferroptosis ${ }^{23-25}$. Ferritin is a hetero-polymeric molecule composed of 24 heavy and light subunits which can store excess iron in cells of up to 4500 iron atoms ${ }^{26}$. Ferritin is composed of varying proportions of two gene products, heavy ferritin (FTH1) and light ferritin (FTL). The FTH1 exhibits ferroxidase activity which converts toxic ferrous ions into ferric ions, while the light subunit facilitates iron storage ${ }^{27-29}$. Given the role of $\mathrm{CPX}$ as an iron chelator and the role in multiple pathways shown to be altered in PKD, we hypothesized that CPX-O ameliorates disease progression in PKD by mechanisms that involve iron-ferritin axis. 
Our data indicate that CPX-O can reduce cyst formation in 3D collagen assays from primary cystic epithelial cells from ADPKD patients. CPX-O can also attenuate disease progression in a PKD mouse model. Mechanistically, CPX-O appeared to reduce the elevated ferritin expression in cystic epithelial cells by the process of ferritinophagy both in vivo and in vitro.

\section{Results}

\section{CPX-O inhibits cyst formation of ADPKD cells in 3D cyst assays}

To first validate whether CPX-O may be a drug of choice for PKD in the clinics, and whether further mouse studies are required to validate it, we performed 3D cyst assays using primary ADPKD renal epithelial cells ${ }^{30}$. ADPKD cells were induced to form cysts in a collagen gel in the presence of the cAMP agonist, FSK, and growth factor, EGF. After cyst formation, CPX-O at desired concentrations or vehicle was used for 6 days and the effects of CPX-O on cyst growth were evaluated. As shown in figures $1 \mathrm{~A}$ and $1 \mathrm{~B}$, vehicle alone did not affect cyst size, but concentrations as low as $0.2 \mu \mathrm{M}$ CPX-O significantly $(p<0.05)$ decreased cyst area and further decreased at $0.5 \mu \mathrm{M}$ CPX-O $(P<0.01)$. We have previously shown equivalent fold reduction in cyst size with $50 \mu \mathrm{M}$ DAPT (N-(N-(3,5-Difluorophenacetyl)-S phenylglycine t-butyl ester) treatment ${ }^{31}$. DAPT is a gamma secretase inhibitor widely used to inhibit Notch signaling pathway. CPX-O at the above-mentioned concentrations did not affect viability of NHK or ADPKD cells (supplementary fig. 1). In figure 1C, data from individual patient cells (K268) is shown (in 6 replicates) and cyst size is represented as 
surface area showing similar effect with increasing CPX-O concentrations as in figure1B. The data indicated that CPX-O inhibits cyst formation in ADPKD.

\section{CPX-O attenuates disease progression in a mouse model of ADPKD}

We next examined the effects of CPX-O in vivo in a mouse model of ADPKD. We generated $P k d 1^{R C / R C} / P k d 2^{+/-}$mice (hereafter called PKD mice), which show moderate cystic disease progression from P15-P60 ${ }^{32,33}$, and used wild-type (WT; $\left.P k d 1^{+/+} / P k d 2^{+/+}\right)$as controls. Mice were weaned at post-natal $(\mathrm{P})$ day 21 and daily intraperitoneal injections of CPX-O (10mg/kg body weight) were administered from P22-P49. Mice were euthanized at P50, and tissues and blood samples were collected (Fig. 2A). We did not notice any adverse events with CPX-O treatment at the above dose and mice remained active throughout the duration of the study. Kidneys of vehicle and CPX-O treated WT mice appeared similar. In contrast, a significant reduction in kidney size (fig. 2B) and cystic index (fig. 2C) was observed in PKD mice treated with CPX-O compared to those with vehicle. Renal function, as measured by blood urea nitrogen levels, was also statistically different between groups (fig.2D). As expected, the kidney size correlated with kidney to body weight ratio (fig. 2E) showing a significant reduction $(p<0.05)$ in CPX-O treated PKD group compared to vehicle treated PKD group. The data clearly showed a protective effect of CPX-O on the progression of PKD.

\section{Protection mediated by CPX-O does not involve Notch pathway inhibition}


We have previously shown that the Notch3 signaling pathway was activated in human ADPKD cyst lining cells ${ }^{31}$. The Notch pathway is activated when Notch receptors, Notch1, Notch2, Notch3 or Notch4 bind to their ligands. Binding of the receptor to the ligand results in the proteolytic cleavage mediated by the Gamma secretase complex. This results in the release of the Notch intracellular domain (NICD) and its translocation into the nucleus. In the nucleus, the NICD binds to CSL/RBPjk protein to activate the downstream targets of Notch signaling such as Hes1. We analyzed Notch3 pathway using kidney lysates and kidney sections from vehicle or CPX-O treated WT or PKD groups. Notch3, as shown previously, was upregulated in the PKD sections (fig. 3A), however, CPX-O treatments did not appear to alter the Notch3 expression; this was further verified by Western blots (WB) to detect Notch 3 intracellular domain (N3IC) (fig. 3B, C). Likewise, other Notch signaling members, Hes1 and RBPjk increased in vehicle treated PKD kidneys compared to vehicle treated WT kidneys but the expression remained unaltered with CPX-O (fig. 3B, D and E). The data was confirmed by quantitative analysis of the Western blots normalized with total protein (fig. 3C, D and $\mathrm{E})$.

\section{CPX-O reduces cell proliferation and fibrosis in PKD}

To determine whether CPX-O mediates affect on cell proliferation, kidney sections were stained for Ki67 antigen and couter stained with hematoxylin to count total cells. Ki67 positive cells were clearly seen in abundance in the cystic epithelia (Fig. 4a, arrow). Figure 4B represents quantitative analysis where Ki67 
labeling is presented as percent positive cells per section ( $n=17$ each group), which represented three mice in each group. In addition, Mason trichrome staining and alpha smooth muscle actin labeling was less intense in CPX-O treated PKD sections compared to vehicle treated PKD sections, suggesting a decrease in fibrosis (supplementary fig. 2).

\section{CPX-O inhibits ferritin accumulation in the cystic and interstitial compartments in ADPKD Kidneys}

Next, we examined potential mechanisms by which CPX-O could reduce cystogenesis. CPX-O is an iron chelator, and ferritin degradation has been shown as a mechanism of CPX-O ${ }^{22}$. Immunohistochemistry for ferritin revealed a marked increase in ferritin expression in vehicle treated PKD sections versus WT. Ferritin was localized in the tubular epithelial cells as well as in the interstitial cells. CPX-O decreased overall ferritin staining in PKD mice and in WT mice as observed by IHC (fig.5A). Western blot (WB) analysis of vehicle and CPX-O treated lysates also showed a significant increase in ferritin expression in lysates of vehicle-treated PKD mice compared to vehicle-treated WT mice $(p<0.01)$. Moreover, ferritin was reduced significantly $(p<0.05)$ in renal lysates of CPX- O treated PKD mice (fig. 5B).

To evaluate ferritin status in PKD patients, we examined ferritin expression in normal human kidneys verses PKD kidneys. Primary NHK (normal human kidney) and ADPKD cells were grown in culture under normal conditions and lysed for WB detection of ferritin (fig. 5C). A clear upregulation of heavy chain 
ferritin was observed $(p<0.001)(n=3)$. Next, we tested ferritin in human PKD verses normal kidney (control) via IHC. Ferritin was found in tubule cells and to a lesser extent in the interstitial compartment of normal kidneys, and it was highly expressed in the DBA-positive cysts (fig. 5D). Ferritin is a ubiquitous protein and has been shown to be localized in proximal tubular cells, however its expression in collecting duct cells is not well established. We show that ferritin (fig. $5 \mathrm{E}, \mathrm{H}$ ) (heavy chain, green) is mildly expressed in collecting ducts (DBA positive, red) (fig. 5F, H) of adult human kidney (control), however in the context of PKD, ferritin expression is upregulated in collecting duct cells (lower panels, fig. (5E, F, H). In addition, ferritin expression is upregulated in few interstitial cells (arrow, fig. $5 \mathrm{H}$ lower panel) in PKD.

\section{CPX-O induces ferritinophagy to normalize ferritin expression in PKD}

Ferritin degradation via autophagy has been a proposed mechanism of action of CPX-O ${ }^{11,22}$. Autophagy is a process that results in the formation of autophagosomes in which double membraned vesicular structures sequester cytoplasmic components and fuse with lysosomes to form autolysosomes. In these structures, engulfed cargo is broken down by lysosome-derived acid hydrolases. When nuclear receptor coactivator 4 (NCOA 4), a cargo receptor, binds to ferritin, it targets ferritin degradation, a process that is termed" ferritinophagy" ${ }^{34-38}$. We determined whether ferritin reduction in CPX-O treated PKD mice involved autophagy and more specifically ferritinophagy. WB analysis in kidney lysates from mice revealed that an autophagy marker, LC3B was 
downregulated in vehicle-treated PKD samples $(n=4)$ relative to vehicle-treated WT samples ( $n=3$ ) (Fig. 6A). Interestingly, CPX-O was able to clearly upregulate LC3B-II levels in CPX- O treated PKD samples compared to vehicle-treated PKD samples, whereas CPX-O did not appear have a difference in the WT kidneys. These data were similar as NCOA4 expression, which was downregulated in PKD indicating reduced ferritin degradation and reversal of this phenomenon by CPX-O and hence upregulated NCOA4. This process was also observed in wildtype CPX-O treated mouse kidney lysates however, the ferritin staining was weaker compared to kidneys from PKD mice.

To determine whether the induced ferritinophagy is a direct effect of CPX-O, we used ADPKD primary human cells to look for a dose dependent response on ferritin and NCOA 4. As expected, (figure 6B), ferritin expression decreased with increasing concentrations of CPX-O. This was also corroborated using Immunocytochemistry (fig. 6C). ADPKD cells expressed more ferritin (green) than NHK cells, which localized to both nucleus and cytoplasm and was clearly visible in cytoplasm in ADPKD cells (arrows) (fig.6C). With increasing CPX-O concentrations, ferritin expression in the cytoplasm diminished (arrows, fig 6C). The nuclear labeling in primary cells appeared to be only a property of cells in culture because such effect was not seen in human or mouse kidneys. These data indicate that ferritinophagy, as demonstrated by the loss of ferritin, is accompanied by an increase in NCOA4.

\section{Discussion}


To our knowledge, we report for the first time that ferritin is upregulated in renal cells in PKD, and CPX-O normalizes the ferritin levels and protects against cyst progression in ADPKD.

In the kidneys, proximal tubules are the major source of heavy chain ferritin (FTH1) expression ${ }^{39}$. The current study with human primary cells shows ferritin upregulation in cystic cells which are mainly of collecting duct origin in ADPKD kidneys. Also DBA positive cells in PKD kidneys, stained positive for ferritin, indicating that under pathologic circumstances, ferritin is increased in the cystic cells, similar to that reported in cancer and other pathologic diseases ${ }^{40-44}$.

Conditional knock down of proximal tubule specific FTH1 in mice worsened acute kidney injury and was associated with increased apoptosis and significant mortality. The study indicated a protective role of FTH1 in proximal tubules in AKI 39. On the contrary, deletion of myeloid FTH1 was protective against lipopolysaccharide induced endotoxemia in mice. In this model compensatory increased in circulating FTL was reported ${ }^{45}$. In addition, there are reports on iron independent roles of ferritin ${ }^{44}$. Ferritin levels in serum correlate with the body's iron stores, however under inflammatory conditions such as cancer or autoimmune diseases, elevated ferritin levels are reported which do not correlate with the body's iron levels ${ }^{43}$. In breast cancer cells, the binding and uptake of ferritin was observed along with increased cell growth independent of the iron status of ferritin ${ }^{44}$. Our data suggest that in PKD ferritin upregulation is a 
pathogenic response, whether it is iron dependent or independent would need further studies.

Ferritin treatment on endothelial cells promoted activity of Erk and AKT signaling promoting pro- angiogenic effects ${ }^{41}$. These pro-survival pathways are also activated in ADPKD cells and thus it is tempting to speculate that ferritin accumulation may play a role in elevating these pathways as well as other pathways that are activated in PKD ${ }^{3}$. In our studies, the Notch 3 pathway is activated in PKD tissues which correlated with the ferritin increase, but CPX-O $(10 \mathrm{mg} / \mathrm{ml}$ body weight) mediated ferritin reduction did not correlate with the Notch 3 pathway which indicates that in kidney, CPX-O does not alter Notch 3 signaling pathway at the low dose used in our study.

In recent years, CPX-O has emerged as an important anticancer agent and is currently in clinical trials for advanced hematological malignancies ${ }^{19}$. The protective effects of CPX-O have been attributed to its potential to chelate iron and thus effect iron dependent pathways and enzymes, which may potentially lead to inhibition of cell proliferation and induction of cell death in tumors ${ }^{13}$. Iron is an essential element in the body and also regulates the heme pathway. Unbound intracellular iron generates reactive oxygen species (ROS) through Fenton chemistry leading to DNA breaks, lipid peroxidation, and cellular damage. Thus, a tight regulation of iron metabolism is required to maintain cellular homeostasis. However it is known that CPX-O is a mild iron chelator and its use in small doses does not appear to affect the heme group, instead it was recently 
suggested that CPX can stabilize heme groups instead of competing with them for metal chelation ${ }^{46}$.

In our studies we find that ADPKD cells are more sensitive to CPX-O than the NHK cells. This was shown by the dose response curve in supplementary data where NHK cells resisted cell death upto $10 \mu \mathrm{M}$ concentration, whereas ADPKD cells responded to $1 \mu \mathrm{M}$ or less. In $3 \mathrm{D}$ collagen assays, only 0.2 to $0.5 \mu \mathrm{M}$ was enough to significantly inhibit cyst growth. In mouse studies we used only $10 \mathrm{mg} / \mathrm{g}$ body weight dose which was sufficient to reduce cystogenesis and normalize kidney function in PKD mice. Our studies suggest that low doses of CPX-O are enough for the cystic cells to respond.

The autophagic degradation of ferritin, an iron storage protein has been reported to be a key mechanism maintaining iron homeostasis ${ }^{38,47}$. We show that ferritin expression is increased in cyst lining epithelial cells and CPX-O can induce ferritinophagy in these cells. The process of ferritinophagy involves release free iron and resulting in ferroptosis (iron induced cell death), which could be detrimental for cells, a possibility is that CPX-O provides dual protection in PKD by inducing ferritinophagy and protecting against ferroptosis as described previously ${ }^{24}$. Whether CPX-O results in protection against ferroptosis in PKD will require further investigation.

This study opens avenues for future studies regarding the role of iron metabolism in PKD. Available literature on extracellular ferritin studies shows that ADPKD patients have higher levels of hemoglobin compared to other forms of CKD 
patients. This has been thought to be a consequence of increased erythropoietin production by cystic cells ${ }^{48,49}$. Average ferritin levels of $100-800 \mathrm{ng} / \mathrm{mL}$ was associated with the best survival in PKD patients, whereas that of non-PKD patients was $500-800 \mathrm{ng} / \mathrm{mL}$, showing that iron may play a role in PKD ${ }^{50}$. Sequestration of intracellular iron was speculated as a mechanism for cyst reduction by NGAL (Neutrophil gelatinase-associated lipocalin ${ }^{51}$. The role of iron hemeostasis in PKD warrant investigations. However, in the present form, our studies indicate that CPX-O can ameliorate disease progression in PKD, and this process involves induction of ferritinophagy as a mechanism.

\section{Materials and methods}

Antibodies and reagents: Antibodies and their sources are listed: LC3B and Ferritin from Abcam (Cambridge, MA), NCOA4 and smooth muscle actin from (Sigma Aldrich, Saint Louis, MO), RBPjk and Hes1 (Santa Cruz, Dallas, TX), Presenilin1 (Genscript, Piscataway, NJ), HeyL (Life Span Biosciences, Seattle, WA). Ki67 from Dako, DAPT (N-[N-(3,5-Difluorophenacetyl)-L-alanyl]-Sphenylglycine t-butyl ester) was obtained from Apex Biosciences ( Chapel Hill, NC). Ciclopirox olamine (CPX-O) from Sigma Aldrich, Saint Louis, MO.

Animal care and protocol: All mice were maintained in accordance with the recommendations in the Guide for the Care and Use of Laboratory Animals of the National Institutes of Health. The protocol was approved by the University of Kansas Medical Center Institutional Animal Care and Use Committee on the ethics of animal experiments. $P k d 1^{R C / R C}: P k d 2^{+/-}(\mathrm{PKD})$ mice were generated by 
breeding $P k d 1^{R C / R C}$ mice with $P k d 2^{+-}$mice kindly provided by Drs. Peter Harris and Steven Somlo, respectively ${ }^{32,33}$. Homozygous $P k d 1^{R C / R C}$ and heterozygous $P k d 2^{+/-}$mice have a slow progressing cyst phenotype. However, when these mice are bred to obtain $P K D 1^{R C / R C}, P K D 2^{+/-}$mice, cyst formation is accelerated. Wildtype littermates without a PKD mutation $\left(P k d 1^{+/ / /} / P k d 2^{+/+}\right)$were used as controls. The $P K D 1^{1 R C / R C}, P K D 2^{+/}(\mathrm{PKD})$ mice are mildly cystic at birth and the cysts progress with age such that there is an exponential growth of the cysts between age P15 and P60 after which cyst growth does not advance. We used these mice because the time period of cyst formation was moderate for the drug studies.

CPX-O Treatments in mice: CPX-O was solubilized in 0.5M phosphate buffer $\mathrm{pH} 9.5$ and filter sterilized using a 0.2 micron filter for in vivo treatments. 12 PKD and 12WT mice (males and females) were weaned and treatments were started at postnatal day 22 for a total of 27 days with daily intraperitoneal injections of $10 \mathrm{mg} / \mathrm{kg}$ body weight CPX-O (fig. 2). Mice were obtained from the PKD rodent core facility at the Kansas University Medical Center. Six mice from each group (WT and PKD) received vehicle alone and 6 received CPX-O. During euthanasia, mice were weighed and perfused with cold PBS after blood collection followed by collecting and weighing kidneys. One kidney was snap frozen and the other was fixed in $4 \%$ paraformaldehyde for $24 \mathrm{hrs}$ followed by storage in $70 \%$ ethanol at $4^{\circ} \mathrm{C}$ until blocking and sectioning for histology and immunohistochemistry. 
Histology, cystic Index and blood urea nitrogen (BUN) measurements: The fixed kidney tissues were processed and embedded in paraffin at the core facilities of the University of Kansas Medical Center. Five-micrometer sections were stained with Hematoxylin and Eosin (H\&E) as described previously 52. Cystic index was measured using ImageJ on H\&E stained kidney sections. The area of each individual cyst within the section of entire kidney was calculated and then added together. This summed value was then divided by the total area of the section yielding in the value identified as the cystic index. This was done for a kidney section for each mouse used in the study $(n=6)$ in each PKD group. BUN was quantified using a Quantichrom Urea Assay Kit (Bioassay Systems), according to the manufacturer's protocol.

Human cells and tissues: ADPKD kidneys were obtained from the surgery department at KUMC with the assistance of the KU Cancer Center's Biospecimen Resource Core and hospitals participating in the Tissue Donation Program at the PKD Foundation (Kansas City, MO). NHK tissues were obtained from nephrectomy specimens by the surgery department at KUMC. Primary cultures of ADPKD and NHK epithelial cells were generated as described previously ${ }^{53}$. Use of de-identified surgically discarded tissues complies with federal regulations and was approved by the Institutional Review Board at KUMC. ADPKD cells were obtained from multiple surface cysts ranging in size. NHK cells were cultured from sections of cortex. These cells have been shown to be enriched in collecting duct marker, Dolichos biflorus agglutinin (DBA) ${ }^{3}$. Cells were cultured in DMEM/F-12 supplemented with $5 \% \mathrm{FBS}, 5 \mu \mathrm{g} / \mathrm{ml}$ insulin, $5 \mu \mathrm{g} / \mathrm{ml}$ 
transferrin, and $5 \mathrm{ng} / \mathrm{ml}$ sodium selenite (ITS, Thermo Scientific) and penicillin $(100 \mathrm{U} / \mathrm{ml})$, streptomycin $(130 \mu \mathrm{g} / \mathrm{ml})(\text { Pen/Strep })^{54}$. Cultures were not passaged more than twice before being used in experiments. Cells were grown in an incubator at $37^{\circ} \mathrm{C}$ under $5 \% \mathrm{CO}_{2}$.

Cell culture and treatments: NHK cells and ADPKD cells were grown to $80 \%$ confluency in a $10 \mathrm{~mm}$ cell culture dish. Cells were washed and lysed with RIPA lysis buffer (50 mM Tris $\mathrm{HCl}$ pH7.5, $137 \mathrm{mM} \mathrm{NaCl}, 1 \%$ IGEPAL, $2 \mathrm{mM}$ EDTA), and Complete Protease Inhibitor (Thermo scientific) for Western blots. For treatments, ADPKD cells were grown to $70 \%$ confluency followed by a 24 -hour low serum $(0.001 \%$ and no ITS) treatment. Cells were then treated with vehicle or CPX-O for 24 hours before lysis.

Cell viability assay: ADPKD or NHK cells were plated in a 12 well plate $(20,000$ cells/well) and allowed to grow overnight. The following morning cells were treated with either vehicle control $(0.5 \mathrm{M}$ phosphate buffer $\mathrm{pH} 9.5)$ or increasing concentration of CPX-O. After 24 hours of incubation at $37^{\circ} \mathrm{C}$, and $5 \% \mathrm{CO}_{2}$, cells were trypsinized and pelleted. Cells were suspended in 500ul media and cell viability was tested in triplicates using Cell counting kit-8 (CCK-8, Apex Biosciences, Houston, TX) and manufacturer's instructions were followed. Viability was set at $100 \%$ for the vehicle control and relative values were calculated for other doses.

In vitro 3D cyst assays: In vitro cyst assays were performed as described 6 30,55. Briefly, primary cultures of ADPKD cells were suspended in media containing 
type 1 collagen (PureCol, Advanced biomatrix, San Diego, CA) in a 96-well plate. Immediately after adding collagen and cells $\left(4 \times 10^{3} / 100 \mathrm{ul}\right), 100 \mathrm{ul}$ of media with collagen and cells was pipetted into each well of the 96-well plate. The plate was incubated at $37^{\circ} \mathrm{C}$ for 45 minutes to allow collagen to polymerize. Then, $150 \mu$ l of defined media (1:1 DMEM/F12 with ITS, $5 \times 10^{-8} \mathrm{M}$ hydrocortisone, $5 \times 10^{-5} \mathrm{M}$ triiodothyronine) containing $5 \mu \mathrm{M}$ forskolin and $5 \mathrm{ng} / \mathrm{ml}$ EGF was added on to the polymerized gel to initiate cyst growth. Following cyst growth between day 5 to 6 , the agonists (FSK and EGF) were removed and the gels were rinsed twice with defined media. To initiate drug treatments, CPX-O at different concentrations was added to the wells. For control, media containing $50 \mu \mathrm{M}$ DAPT (N-(N-(3,5-Difluorophenacetyl)-S phenylglycine t-butyl ester) was added. Fresh treatment media was replaced every day for each treatment. After 5-7 days, the outer diameter of cross-sectional images of spherical cysts with distinct lumens were measured using a digital camera attached to an inverted microscope and analyzed with video analysis software (image pro-premier). Surface area was calculated from the outer diameters and total surface area of the cysts was determined from the sum of individual cysts within each well. Cysts with diameters of $50 \mu \mathrm{M}$ or less were excluded. Data is presented as surface area $/ \mu m^{2}$ or surface area fold change. Experiments were replicated six times from at least three different ADPKD patients.

Immuno-histochemistry (IHC)/ Immunofluorescence (IF): IHC was performed as described previously ${ }^{56}$. Briefly, kidney sections from wild type and PKD mice treated with CPX-O or vehicle (veh) were deparaffinized with Xylene and 
hydrated with graded ethanols. Sections were then boiled in citrate buffer (10 mM sodium citrate, $0.05 \%$ tween $20, \mathrm{pH}: 6.0$ ) and cooled to room temperature. Sections were incubated for 30 min with $3 \%$ hydrogen peroxide for $\mathrm{IHC}$ or $0.5 \mathrm{M}$ ammonium chloride for IF to block endogenous peroxidase or fluorescence activity, respectively. Subsequent washing in PBS and blocking with $10 \%$ normal serum (in PBS from the species the secondary antibody was raised in) for $1 \mathrm{~h}$ were followed by incubation for $1 \mathrm{~h}$ with primary antibodies in a humidified chamber. Slides were washed three times in PBS and incubated for $1 \mathrm{~h}$ in 1:400 diluted biotin-conjugated secondary antibodies (Vector Laboratories, Burlingame, CA) for IHC and 1:400 fluorescein/Texas red-conjugated antibodies for IF. Slides were washed four times in PBS. For IF, the slides were cover slipped using Vecta shield with DAPI (Vector Labs). For IHC, the slides were further incubated with avidin-biotin-peroxidase complex (ABC Elite; Vector Laboratories, Burlingame, CA) and detected with diaminobenzidine (DAB; Sigma Aldrich, St. Louis, MO). Tissue sections for IHC were then dehydrated with graded ethanol and mounted with permount (Fisher Scientific, Pittsburg, PA). Slides were viewed on a Leica DMR microscope.

Immunocytochemistry (ICC): Immunocytochemistry was performed in NHK and ADPKD cells grown in 8-well chamber slides to $80 \%$ confluency and treated with vehicle or increasing concentrations of CPX-O for $24 \mathrm{hrs}$. The cells were fixed in $4 \%$ paraformaldehyde for fifteen minutes followed by incubation with $100 \mathrm{mM}$ ammonium chloride for five minutes twice. Cells were then permeabilized with $0.05 \%$ Triton in PBS for five minutes. The slides were blocked with 10\% BSA for 
1hr. Primary antibody and secondary antibody and washing steps were same as described above for IF. After mounting with Vecta shield containing DAPI (Vector Laboratories), slides were sealed with nail liquor and analyzed under a Leica DMR microscope.

Western blots: Following treatments, cells were washed with PBS three times and lysed. Tissues (fresh or frozen) were chopped in pieces and homogenized using a Dounce homogenizer. For both cells and tissues, RIPA lysis buffer (50 mM Tris $\mathrm{HCl} \mathrm{pH} 7.5,137 \mathrm{mM} \mathrm{NaCl}, 1 \%$ IGEPAL, $2 \mathrm{mM}$ EDTA) with protease inhibitors (Protease inhibitor cocktail, Fisher Scientific, Pittsburg, PA) was used ${ }^{31}$. Protein concentration was measured using BCA protein assay (Bio-Rad, Hercules, CA). Whole cell lysates (50 to $100 \mu \mathrm{g}$ ) were solubilized in $6 \mathrm{X}$ Laemmli sample buffer and heated to $95^{\circ} \mathrm{C}$ for ten minutes and electrophoresed on $15 \%$ (for ferritin and LC3B) and 10\% (for other proteins) polyacrylamide gels. Proteins were transferred to PVDF membranes. Ponceau staining was performed for each blot to determine protein transfer and imaged. Ponceau S (total protein was used to normalize the gels for protein loading ${ }^{57}$ ). The immunoblots were blocked in $5 \%$ nonfat dry milk in PBST (PBS containing $0.1 \%$ Tween 20) for 1 hour at room temperature and then followed by PBS washes; the blots were incubated with appropriate dilutions of primary antibodies overnight. The blots were then washed and incubated with secondary antibodies (1:10,000 dilution in blocking solution) for 1 hour at room temperature. After subsequent washes in PBST, bound antibody was detected by chemiluminescence (Western Lightning Plus ECL, Perkin Elmer). Bands produced in the results were quantified using image 
and normalized with ponceau S staining to confirm equal loading ${ }^{57}$. Data was presented as relative fold change.

\section{Statistics}

Data are expressed as mean \pm SE. Statistical significance was measured by Student's unpaired T test for comparison between control and PKD groups. Oneway ANOVA was performed to compare more than two groups followed by Tukey HSD test. A $P$ value of $<0.05$ was considered statistically significant.

\section{Disclosure}

The authors have no disclosures.

\section{Acknowledgements}

We thank Dr. Alan Yu for his constructive comments on the project and his help editing the final draft of the manuscript. We thank the rodent core individuals, Dr. Steven Parnell and Heather Riddle for help generating and genotyping the mice, Sireesha Yerrathota for technical help. Gail Reif, Yan Zhang and Emily Daniel for help with obtaining NHK and ADPKD cells. 


\section{References}

1. Chapin HC, Caplan MJ. The cell biology of polycystic kidney disease. $J$ Cell Biol. 2010;191(4):701-710.

2. Ibraghimov-Beskrovnaya $\mathrm{O}$, Natoli TA. mTOR signaling in polycystic kidney disease. Trends Mol Med. 2011;17(11):625-633.

3. Yamaguchi T, Nagao S, Wallace DP, et al. Cyclic AMP activates B-Raf and ERK in cyst epithelial cells from autosomal-dominant polycystic kidneys. Kidney Int. 2003;63(6):1983-1994.

4. Yamaguchi T, Wallace DP, Magenheimer BS, Hempson SJ, Grantham JJ, Calvet JP. Calcium restriction allows cAMP activation of the B-Raf/ERK pathway, switching cells to a cAMP-dependent growth-stimulated phenotype. J Biol Chem. 2004;279(39):40419-40430.

5. Mangoo-Karim R, Ye M, Wallace DP, Grantham JJ, Sullivan LP. Anion secretion drives fluid secretion by monolayers of cultured human polycystic cells. Am J Physiol renal Physiol. 1995;269:F381-388.

6. Reif GA, Yamaguchi T, Nivens E, Fujiki H, Pinto CS, Wallace DP. Tolvaptan inhibits ERK-dependent cell proliferation, $\mathrm{Cl}^{-}$secretion, and in vitro cyst growth of human ADPKD cells stimulated by vasopressin. Am J Physiol Renal Physiol. 2011;301:F1005-1013.

7. Benzing $\mathrm{T}$, Simons $\mathrm{M}$, Walz G. Wnt signaling in polycystic kidney disease. J Am Soc Nephrol. 2007;18(5):1389-1398.

8. Idowu J, Home T, Patel N, et al. Aberrant Regulation of Notch3 Signaling Pathway in Polycystic Kidney Disease. Scientific reports. 2018;8(1):3340. 
9. Weir SJ, Wood R, Schorno K, et al. Preclinical Pharmacokinetics of Fosciclopirox, a Novel Treatment of Urothelial Cancers, in Rats and Dogs. J Pharmacol Exp Ther. 2019;370(2):148-159.

10. Song S, Christova T, Perusini S, et al. Wnt inhibitor screen reveals iron dependence of $\beta$-catenin signaling in cancers. Cancer Res. $2011 ; 71(24): 7628-7639$.

11. Zhou H, Shen T, Shang C, et al. Ciclopirox induces autophagy through reactive oxygen species-mediated activation of JNK signaling pathway. Oncotarget. 2014;5(20):10140-10150.

12. Zhou H, Shang $\mathrm{C}$, Wang $\mathrm{M}$, et al. Ciclopirox olamine inhibits mTORC1 signaling by activation of AMPK. Biochem Pharmacol. 2016;15(116):3950.

13. Weir SJ, Wood R, Ham T, et al. Preclinical development of ciclopirox prodrug for the treatment of non-muscle invasive and muscle invasive bladder cancer. 2018;36(15_suppl):e14576-e14576.

14. Subissi A, Monti D, Togni G, Mailland F. Ciclopirox: recent nonclinical and clinical data relevant to its use as a topical antimycotic agent. Drugs. 2010;70(16):2133-2152.

15. Alpermann HG, Schütz E. Studies on the pharmacology and toxicology of ciclopiroxolamine Arzneimittelforschung. 1981;31(8A):1328-1332.

16. Yang J, Milasta S, Hu D, et al. Targeting Histone Demethylases in MYCDriven Neuroblastomas with Ciclopirox. Cancer Res 2017;77(17):46264638. 
17. Kang JA, Kim S, Park M, et al. Ciclopirox inhibits Hepatitis B Virus secretion by blocking capsid assembly. Nature communications. 2019;10(1):2184.

18. Alpermann HG, Schutz E. [Studies on the pharmacology and toxicology of ciclopiroxolamine (author's transl)]. Arzneimittelforschung. $1981 ; 31(8 a): 1328-1332$.

19. Minden MD, Hogge DE, Weir SJ, et al. Oral ciclopirox olamine displays biological activity in a phase I study in patients with advanced hematologic malignancies. Am J Hematol. 2014;89(4):363-368.

20. Wu J, Liu H, Zhang G, et al. Antileukemia Effect of Ciclopirox Olamine Is Mediated by Downregulation of Intracellular Ferritin and Inhibition $\beta$ Catenin-c-Myc Signaling Pathway in Glucocorticoid Resistant T-ALL Cell Lines. PLoS One. 2016;11(8):e0161509.

21. Li X, Liu Y, Zheng Q, et al. Ferritin light chain interacts with PEN-2 and affects Y-secretase activity. Neurosci Lett 2013;548(26):90-94.

22. Wu J, Liu H, Zhang G, et al. Antileukemia Effect of Ciclopirox Olamine Is Mediated by Downregulation of Intracellular Ferritin and Inhibition $\beta$ Catenin-c-Myc Signaling Pathway in Glucocorticoid Resistant T-ALL Cell Lines. PLoS One. 2016;11(8):e0161509.

23. Hayano M, Yang WS, Corn CK, Pagano NC, Stockwell BR. Loss of cysteinyl-tRNA synthetase (CARS) induces the transsulfuration pathway and inhibits ferroptosis induced by cystine deprivation. Cell Death Differ 2016;23(2):270-278. 
24. Sun $\mathrm{Y}, \mathrm{Q} B$, Xuan B, et al. Human Cytomegalovirus Protein pUL38 Prevents Premature Cell Death by Binding to Ubiquitin-Specific Protease 24 and Regulating Iron Metabolism. J Virol. 2018;92(13):e00191-00118.

25. De Domenico I, Ward DM, Kaplan J. Specific iron chelators determine the route of ferritin degradation. Blood. 2009;114(2):4546-4551.

26. Harrison PM, Arosio P. The ferritins: molecular properties, iron storage function and cellular regulation. Biochim Biophys Acta. 1996;1275(3):161203.

27. Orino K, Watanabe K. Molecular, physiological and clinical aspects of the iron storage protein ferritin. Vet J. 2008;178(2):191-201.

28. Boyd D, Vecoli C, Belcher DM, Jain SK, Drysdale JW. Structural and functional relationships of human ferritin $\mathrm{H}$ and $\mathrm{L}$ chains deduced from cDNA clones. J Biol Chem. 1985;260(21):11755-11761.

29. Levi S, Yewdall SJ, Harrison PM, et al. Evidence of H- and L-chains have co-operative roles in the iron-uptake mechanism of human ferritin. Biochem J. 1992;288 ( Pt 2):591-596.

30. Sharma M, Reif GA, Wallace DP. In vitro cyst formation of ADPKD cells. Methods Cell Biol. 2019;153:93-111.

31. Idowu J, Home T, Patel N, et al. Aberrant regulation of Notch3 signaling pathway in polycystic kidney disease. Sci Rep. 2018;DOI:

10.1038/s41598-018-21132-3. 
32. Hopp K, Ward CJ, Hommerding CJ, et al. Functional polycystin-1 dosage governs autosomal dominant polycystic kidney disease severity. J Clin Invest. 2012;122(11):4257-4273.

33. Wu G, Markowitz GS, Li L, et al. Cardiac defects and renal failure in mice with targeted mutations in Pkd2. Nat Genet. 2000;24(1):75-78.

34. Tang M, Chen Z, ChenL. Ferritinophagy/ferroptosis: iron related new comers in human diseases. J Cell Physiol. 2018;233(12):1-12.

35. Hou W, Xie $\mathrm{Y}$, Song $\mathrm{X}$, et al. Autophagy promotes ferroptosis by degradation of ferritin. Autophagy 2016;12(8):1425-1428.

36. Gao M, Monian P, Pan Q, Zhang W, Xiang J, Jiang X. Ferroptosis is an autophagic cell death process. Cell Res. 2016;26(9):1021-1032.

37. Shintoku R, Takigawa Y, Yamada K, et al. Lipoxygenase-mediated generation of lipid peroxides enhances ferroptosis induced by erastin and RSL3. Cancer Sci. 2017;108(11):2187-2194.

38. Mancias JD, Wang X, Gygi SP, Harper JW, Kimmelman AC. Quantitative proteomics identifies NCOA4 as the cargo receptor mediating ferritinophagy. Nature 2014;509(7498):105-109.

39. Zarjou A, Bolisetty S, Joseph R, et al. Proximal tubule H-ferritin mediates iron trafficking in acute kidney injury. J Clin Invest. 2013;123(10):44234434.

40. Xu L, Koumenis IL, Tilly JL, Giffard RG. Overexpression of bcl-xL protects astrocytes from glucose deprivation and is associated with higher 
glutathione, ferritin, and iron levels. Anesthesiology. 1999;91(4):10361046.

41. Tesfay L, Huhn AJ, Hatcher H, Torti FM, Torti SV. Ferritin blocks inhibitory effects of two-chain high molecular weight kininogen (HKa) on adhesion and survival signaling in endothelial cells. PLoS One. 2012;7(7):e40030.

42. Bolisetty S, Zarjou A, Hull TD, et al. Macrophage and epithelial cell Hferritin expression regulates renal inflammation. Kidney international. 2015;88(1):95-108.

43. Ahmed A, Alkhateeb, James R. Connor. The significance of ferritin in cancer. Biochim Biophys Acta. 2013;1836:245-254.

44. Alkhateeb AA, Han B, Connor JR. Ferritin stimulates breast cancer cells through an iron-independent mechanism and is localized within tumorassociated macrophages. Breast Cancer Res Treat. 2013;137(3):733-744.

45. Zarjou A, Black LM, McCullough KR, et al. Ferritin Light Chain Confers Protection Against Sepsis-Induced Inflammation and Organ Injury. Front Immunol. 2019;10:131.

46. Urquiza P, Lain A, Sanz-Parra A, et al. Repurposing ciclopirox as a pharmacological chaperone in a model of congenital erythropoietic porphyria. Sci Transl Med. 2018;10(459).

47. Dowdle WE, Nyfeler B, Nagel J, et al. Selective VPS34 inhibitor blocks autophagy and uncovers a role for NCOA4 in ferritin degradation and iron homeostasis in vivo. Nat Cell Biol. 2014;16(11):1069-1079. 
48. Eckardt KU, Möllmann M, Neumann R, et al. Erythropoietin in polycystic kidneys. J Clin Invest. 1989;84(4):1160-1166.

49. Chandra M, Miller ME, Garcia JF, Mossey RT, McVicar M. Serum immunoreactive erythropoietin levels in patients with polycystic kidney disease as compared with other hemodialysis patients. Nephron. 1985;39(1):26-29.

50. Hatamizadeh P, Ravel V, Lukowsky LR, et al. Iron indices and survival in maintenance hemodialysis patients with and without polycystic kidney disease. Nephrol Dial Transplant. 2013;28(11):2889-2898.

51. Wei F, Karihaloo A, Yu Z, et al. Neutrophil gelatinase-associated lipocalin suppresses cyst growth by Pkd1 null cells in vitro and in vivo. Kidney Int. 2013;74(10):1310-1318.

52. Sharma M, Callen S, Zhang D, Singhal PC, Vanden Heuvel GB, Buch S. Activation of Notch signaling pathway in HIV-associated nephropathy. AIDS. 2010;24C(14):2161-2170.

53. Reif GA, Yamaguchi T, Nivens E, Fujiki H, Pinto CS, Wallace DP. Tolvaptan inhibits ERK-dependent cell proliferation, $\mathrm{Cl}(-)$ secretion, and in vitro cyst growth of human ADPKD cells stimulated by vasopressin. Am J Physiol Renal Physiol. 2011;301(5):F1005-1013.

54. Reif GA, Wallace DP. ADPKD cell proliferation and $\mathrm{Cl}(-)$-dependent fluid secretion. Methods Cell Biol. 2019;153:69-92.

55. Wallace DP, Grantham JJ, Sullivan LP. Chloride and fluid secretion by cultured human polycystic kidney cells. Kidney Int. 1996;50(4):1327-1336. 
56. Sharma M, Magenheimer LK, Home T, et al. Inhibition of Notch pathway attenuates the progression of human immunodeficiency virus-associated nephropathy. Am J Physiol Renal Physiol 2013;304(8):F1127-1136.

57. Sander H, Wallace S, Plouse R, Tiwari S, Gomes AV. Ponceau S waste: Ponceau S staining for total protein normalization. Anal Biochem. 2019;575:44-53.

\section{Figure Legends:}

\section{Figure1}

CPX-O inhibits cyst formation of ADPKD cells: ADPKD cells were grown to form cysts on a 3-dimensional system using collagen matrix in the presence of forskolin for 3-5 days. Treatment with vehicle, CPX-O or DAPT was administered for 6 days. (A) Cysts were fixed and imaged. (B) Average fold 
change $(n=4)$ in cyst size with vehicle cyst size set at 1 . (C) Cyst surface area using ADPKD K268 cells. Six replicates/treatment. Statistical significance was determined using one-way ANOVA followed by Tukey's HSD test $(* \mathrm{p}<0.05)$, $* *(\mathrm{p}<0.01)$.

\section{Figure 2}

CPX-O ameliorates disease progression in a mouse model of ADPKD: (A) Experimental timeline where 22-day-old PKD or WT mice were intraperitoneally injected with vehicle (Veh) or CPX-O (10 mg/kg body weight) for 27 consecutive days. The following day, mice were euthanized and samples were collected. (B) H\&E staining of kidneys. Representative images of each treatment group are shown. (C) Renal cystic index of veh and CPX-O treated PKD mice, measured as percent cystic area. (D) Serum urea nitrogen values. (E) Kidney weight to body weight ratio. Data presented as mean +_SE ( $n=4-8$ per group). Statistical significance was determined using unpaired Student's Ttest (C) or one-way ANOVA followed by Tukey's HSD test (D and E) $(* \mathrm{p}<0.05)$.

\section{Figure 3}

Protection mediated by CPX-O does not involve Notch pathway inhibition: (A) Immunohistochemistry (IHC) for Notch3 intracellular domain (N3 IC). (B) Western blot (WB) analysis for Notch3IC, Hes1 and RBPjk (Notch targets) shows increased expression of Notch targets in kidney lysates of vehicle (Veh)treated PKD mice, compared to Veh-treated WT mice. (C, D and E) Protein blots were quantified for N3IC $(n=3)$, Hes1 $(n=5)$ and RBPjk $(n=4)$ using Ponceau S staining for total protein normalization. Statistical significance was determined using One-way ANOVA followed by Tukey's HSD test $(* \mathrm{p}<0.05)$.

\section{Figure 4}

CPX-O slows down cell proliferation in PKD: (A) Immunohistochemistry (IHC) for cell proliferation assessed by Ki67 staining. Arrow points to highly proliferative area on a vehicle (Veh)-treated PKD mouse kidney section. These proliferating cells were less evident in CPX-O treated PKD mice. Hematoxylin staining shows blue nuclei and Ki67 positive nuclei are shown in dark brown and expressed as percent Ki67 positive cells from at least 14 sections per treatment from three different mice per group. Unpaired Student T test used for statistical analysis $(* * \mathrm{p}<0.01)$.

\section{Figure 5}


CPX-O inhibits ferritin accumulation in the cystic and interstitial cells in ADPKD Kidneys: (A) Immunohistochemistry for ferritin in WT and PKD mouse sections treated with vehicle (Veh) or CPX-O. Note accumulation of ferritin-positive cells near cystic area in kidney section of vehicle treated PKD mouse. Ferritin-positive cells were reduced in kidneys of CPX-O treated PKD mice. (B) Western Blot (WB) of kidney lysates for ferritin (left panel) and quantification (right panel) shows increased ferritin in veh-treated PKD mice relative to WT mice and reduced ferritin in CPX-O treated PKD mice relative to veh-treated PKD mice ( $\mathrm{n}=5$-6/group). (C) WB for ferritin using lysates of primary cells from normal or ADPKD patients revealing increased ferritin in $\operatorname{ADPKD}$ ( $\mathrm{n}=3$ patients/group) (left). Quantification is shown in the right panel. (D) Upper panels represent normal human kidney section as control and lower panel represents an ADPKD patient kidney sample with arrows indicating high ferritin intensity both in cyst epithelium and interstitium. (E, F, G and $\mathbf{H})$ Colabelling of ferritin (green) with DBA (a collecting duct marker, red), DAPI (a nuclear stain, blue). Upper panels represents a PKD kidney with no ferritin antibody to serve as a control. Lower panels show ferritin in green. (H) Merged image from panels $\mathrm{E}, \mathrm{F}$ and $\mathrm{G}$ showing ferritin co-localization with DBA (pink) in PKD sections only. One-way ANOVA followed by Tukey's HSD test and unpaired Student T-test were used for statistical analyses of graphs in (B) and $(\mathrm{C})(* \mathrm{p}<0.05, * * \mathrm{p}<0.01, * * * \mathrm{p}<0.001)$.

\section{Figure 6}

CPX-O induces ferritino-phagy in primary cystic epithelial cells from ADPKD patients: (A) Western Blot for autophagy marker, LC3B II, and for ferritinophagy marker, NCOA4, on kidney lysates of WT ( $\mathrm{n}=3$ each) and PKD $(n=4)$ mice treated with vehicle (veh) or CPX. (B) Western blot analysis for ferritin and NCOA4 on lysates of primary cells from ADPKD patients (right panel) treated with varying doses of CPX for $24 \mathrm{hrs}$. CPX treatment reduced ferritin expression and increased NCOA4 expression in a dose-dependent manner. (C) NHK (upper panels) and ADPKD cells (lower panels) were treated with varying doses of CPX-O $(0, .5$ and $1 \mu \mathrm{M})$ for $24 \mathrm{hrs}$. followed by immunolabeling for ferritin (green). ADPKD cells expressed more ferritin than NHK cells and CPX-O dose dependently diminished ferritin expression (arrows) (scale bar: $50 \mu \mathrm{m}$ ). 
A

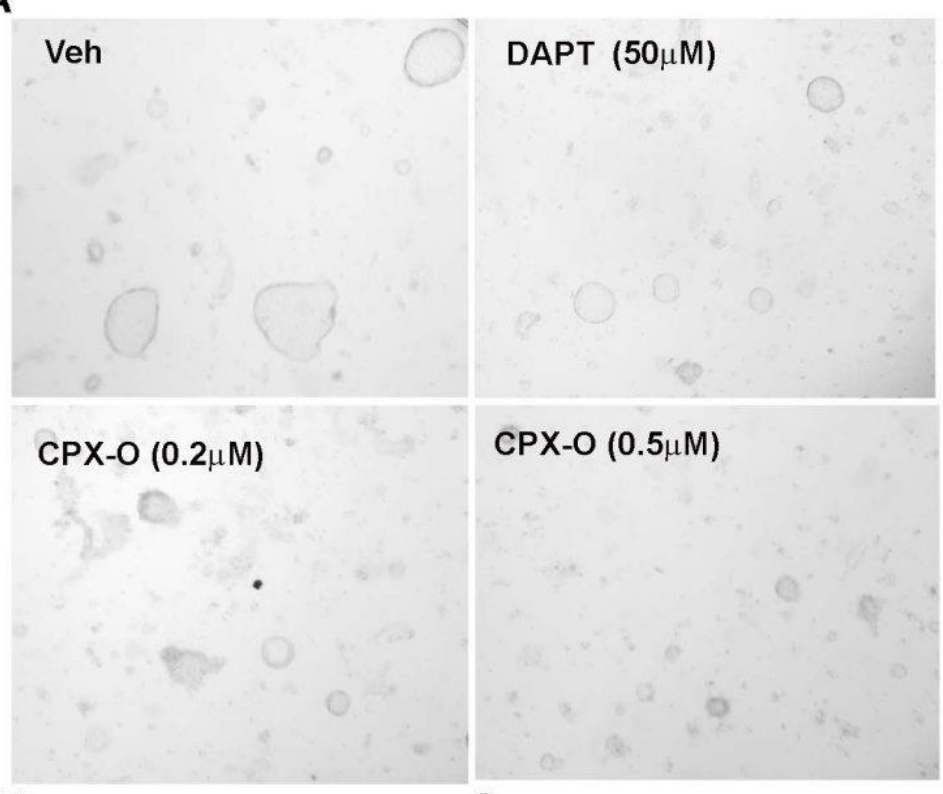

B

C

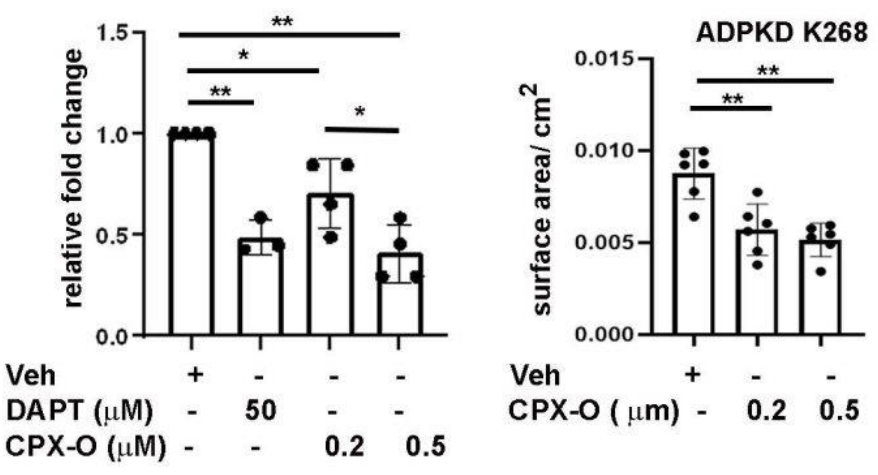


A

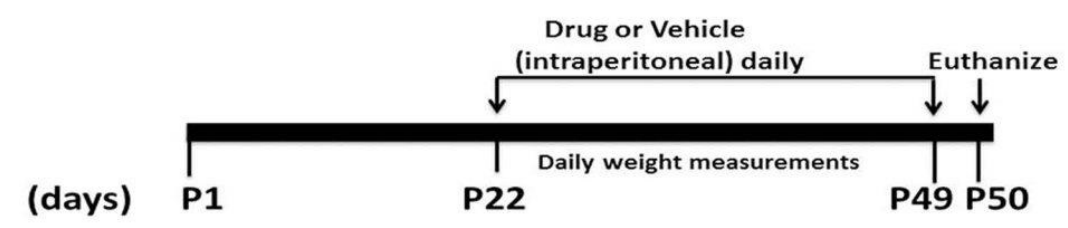

B

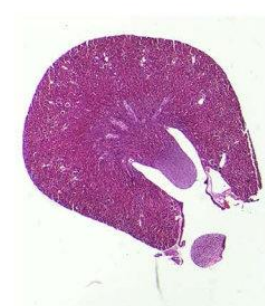

Ciclopiroxolamine: $10 \mathrm{mg} / \mathrm{kg}$ body weight
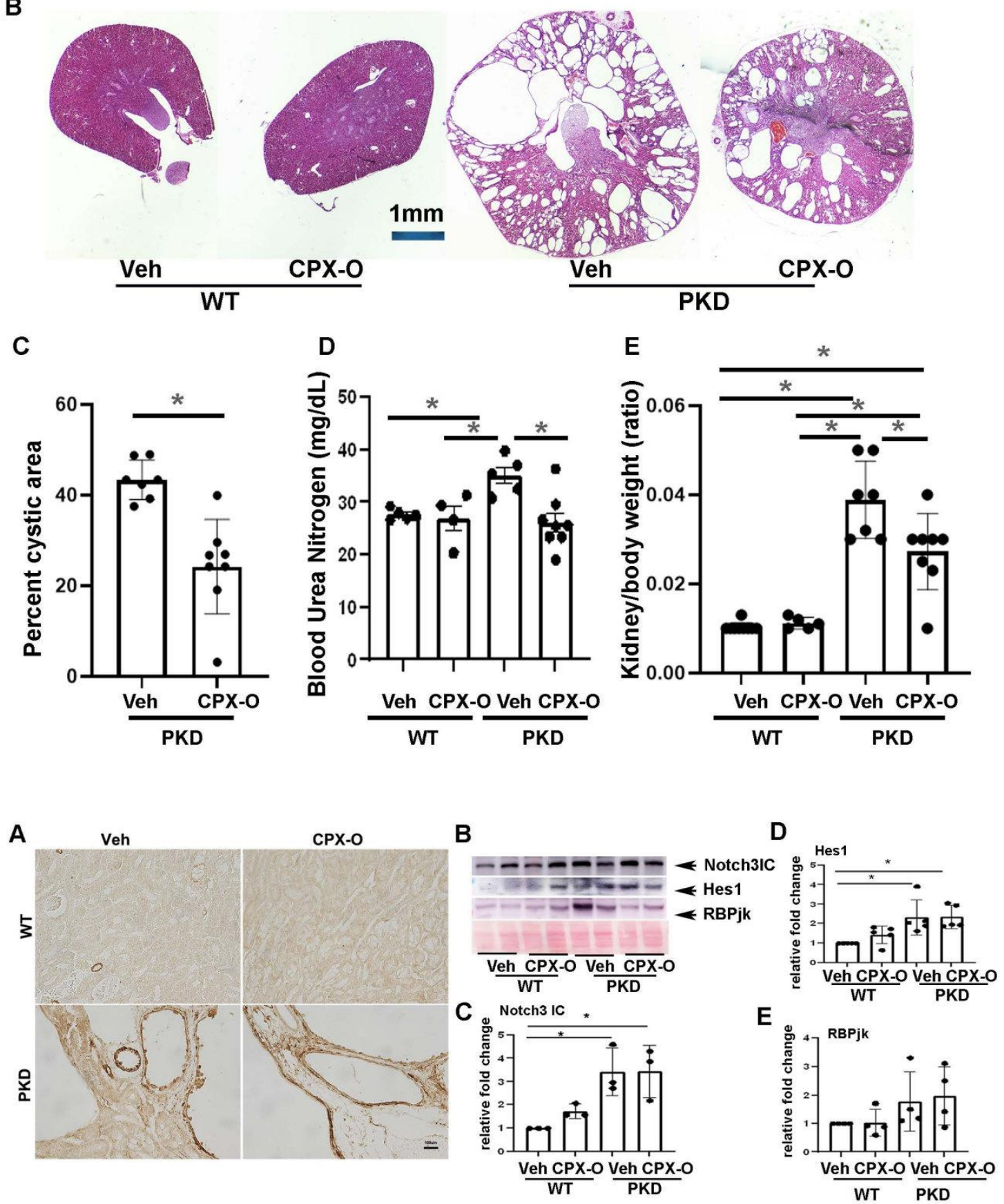

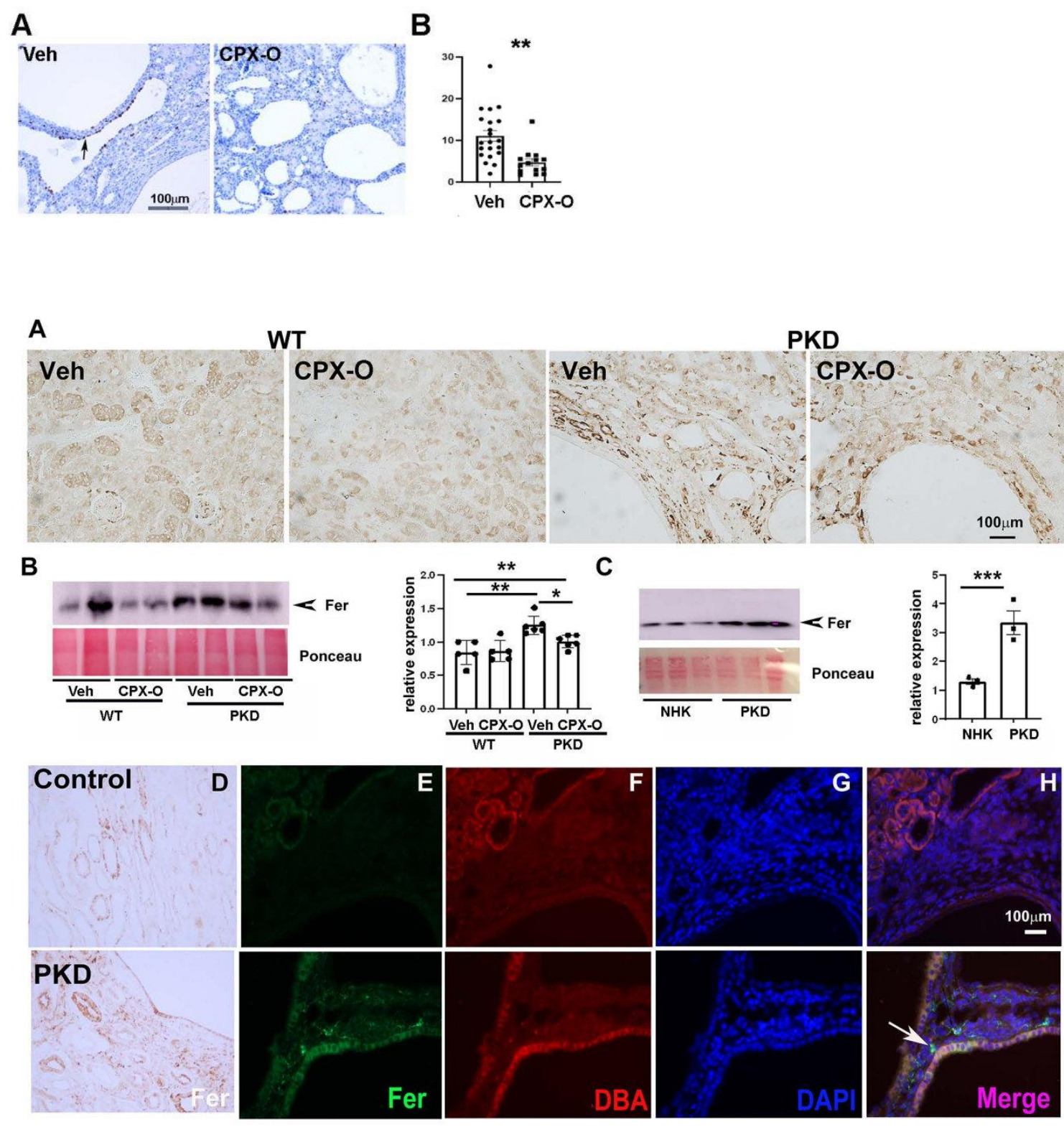
A

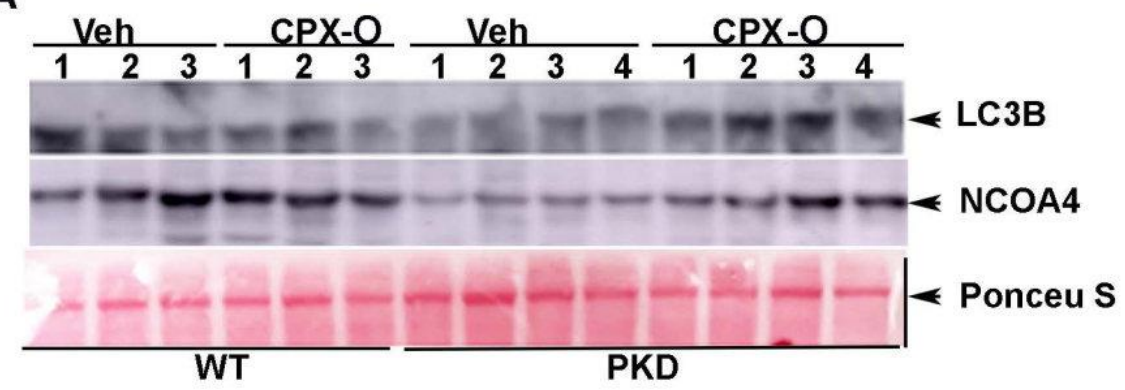

B $\quad \begin{array}{llllll}\text { CPX-O }(\mu \mathrm{M}) & 0 & 1 & 2 & 5\end{array}$

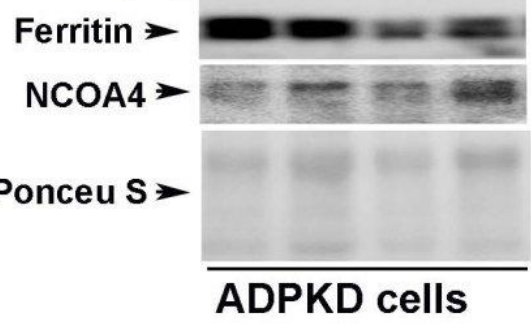

C

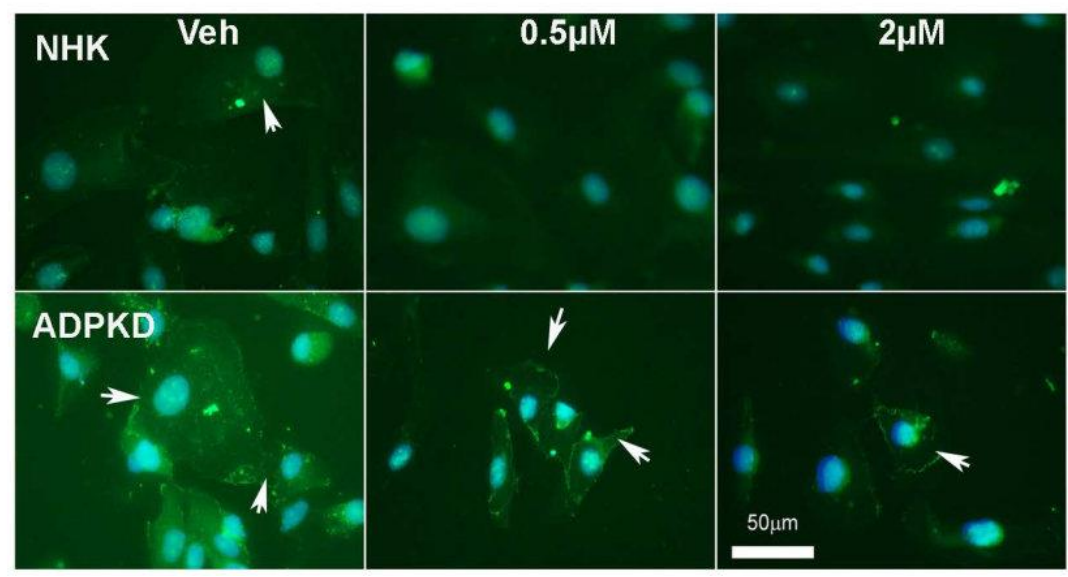

